\title{
Power efficiency and optimum load formulas on RF rectifiers featuring flow-angle equations
}

\author{
Takashi Ohira ${ }^{\text {a) }}$ \\ Toyohashi University of Technology, \\ 1-1 Hibarigaoka, Tempaku, Toyohashi 441-8580 Japan \\ a) ohira@tut.jp
}

\begin{abstract}
This paper considers three pairs of circuit schemes for $\mathrm{RF}$ rectification. Given finite available power from $\mathrm{RF}$ source and load resistance, we formulate DC output power and efficiency of each scheme. Resultant formulas find that load-to-source resistance ratio $R / r$ dominates the circuit performance. Maximum efficiency reaches $81.1 \%$ at $R / r=1$ for single-diode half wave rectifiers. It also reaches $92.3 \%$ for multiple-diode full wave ones at $R / r=1.347$ in bridge with capacitor, 0.742 in bridge with inductor, 5.389 in double-voltage, and 0.1854 in double-current topologies.
\end{abstract}

Keywords: rectifier, power efficiency, optimum load, flow angle

Classification: Microwave and millimeter wave devices, circuits, and systems

\section{References}

[1] M. Saito and K. Araki: Korea Japan Microwave Conference (2011) 58.

[2] T. Saen, K. Itoh, S. Betsudan, S. Makino, T. Hirota, K. Noguchi and M. Shimozawa: IEEE Int. Microwave Workshop Series on Innovative Wireless Power Transmission (2011) 255.

\section{Introduction}

Indeed the rectifier theory looks well-matured, circuit engineers often need numerical simulators in designing radio-frequency $(\mathrm{RF})$ rectifiers. Actually, they have to explore possible topologies and find their optimum load resistance or input matching impedance. This is because RF rectifiers are expected to squeeze DC energy as much as possible from given finite RF energy. Unlike low-frequency power electronics, this requirement is especially crucial for energy harvesting and wireless power transfer applications [1, 2]. This paper considers RF rectifying schemes in single-series, single-shunt, bridge, double-voltage, and double-current topologies. Formulating the circuit behavior from the aspect of diode's flow angle, we derive the optimum load 
condition and maximum available power efficiency for each topology.

\section{RF-to-DC power converter}

Consider an RF rectifier a block having an RF input port and a DC output port as shown in Fig. 1. The RF source with finite available power $P_{\mathrm{s}}$ is represented by time-sinusoidal voltage source

$$
v_{s}(t)=v_{s} \cos \omega t, \quad v_{s}=\sqrt{8 r P_{\mathrm{s}}}, \quad \omega=2 \pi / T
$$

with equivalent internal resistance $r$ in series $[1,2]$. The rectifier receives the $\mathrm{RF}$ power in reduced voltage

$$
v_{1}(t)=v_{s} \cos \omega t-r i_{1}(t)
$$

at its input port, where $i_{1}(t)$ stands for current from the source. On the other side, resistance $R$ simply represents the output load since output voltage $v_{\mathrm{o}}$ and current $i_{\mathrm{o}}$ consist of only their DC components thanks to an ideal smoother we assume inside of the block. Output DC voltage $v_{\mathrm{o}}$, power $P_{\mathrm{o}}$, and power efficiency $\eta$ are therefore written as

$$
v_{\mathrm{o}}=R i_{\mathrm{o}}, \quad P_{\mathrm{o}}=v_{\mathrm{o}} i_{\mathrm{o}}, \quad \eta=\frac{P_{\mathrm{o}}}{P_{\mathrm{s}}}=\frac{8 r}{v_{\mathrm{s}}^{2}} v_{\mathrm{o}} i_{\mathrm{o}}
$$

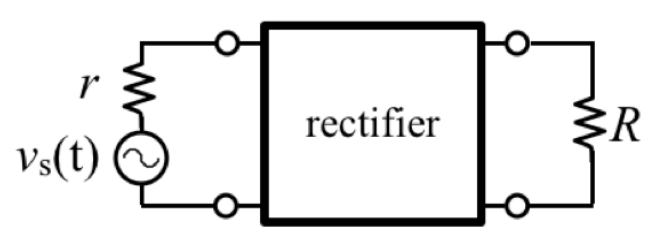

Fig. 1. General RF-to-DC conversion scheme

\section{Single-series topology}

Consider a simple scheme for $\mathrm{RF}$ rectification employing single diode $D$ as shown in Fig. 2. Inductor $L$ makes a DC ground path from the diode to the load, as well as chokes $i_{2}$ against RF current. Namely suppose $\omega L \gg r, R$ to avoid any $\mathrm{RF}$ effect on the input port. Capacitor $C$ makes an $\mathrm{RF}$ ground path from the source to the diode, as well as smoothes out the output ripple. Suppose in the same way $1 / \omega C \ll r, R$ to keep output voltage $v_{\mathrm{o}}$ constant enough at least over time period $T$. For time-invariant load $R$, output current

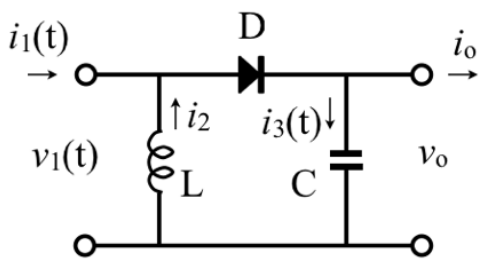

DOI: $10.1587 /$ elex.10.20130230

Received April 02, 2013

Accepted April 25, 2013

Fig. 2. Single-series RF rectifier 
$i_{\mathrm{O}}$ also keeps constant. For simplicity, assume a perfect one-way switch model for diode $D$. Note that $D$ may represent a synchronized switching power transistor.

Let us focus on diode current $i_{\mathrm{d}}$ to satisfy Kirchhoff's current law

$$
i_{1}(t)+i_{2}=i_{d}(t)=i_{3}(t)+i_{\mathrm{o}}
$$

at any time in the period. Note again that $i_{2}$ and $i_{\mathrm{o}}$ are constant as described above. From Eqs. (2) and (4), we get waveforms in two states

(i) $\quad \mathrm{D}=\mathrm{ON} \quad v_{1}(t)=v_{o}, \quad i_{1}(t)=\frac{1}{r}\left(v_{s} \cos \omega t-v_{o}\right) \quad-t_{1}<t<t_{1}$

(ii) $\mathrm{D}=\mathrm{OFF} \quad i_{1}(t)=-i_{2}, \quad v_{1}(t)=v_{s} \cos \omega t+r i_{2} \quad t_{1}<t<T-t_{1}$

which are plotted in Fig. 3. While the RF source provides a pure sinusoidal wave as $v_{\mathrm{S}}(t)$, it is distorted at once for the rectifier input as $v_{1}(t)$. This is due to the strong smoothing capacitor during when the diode makes ON. Integrating Eq. (2) over a period, we get.

$$
\oint v_{1}(t) d t=v_{s} \oint \cos \omega t d t-r \oint i_{1}(t) d t
$$
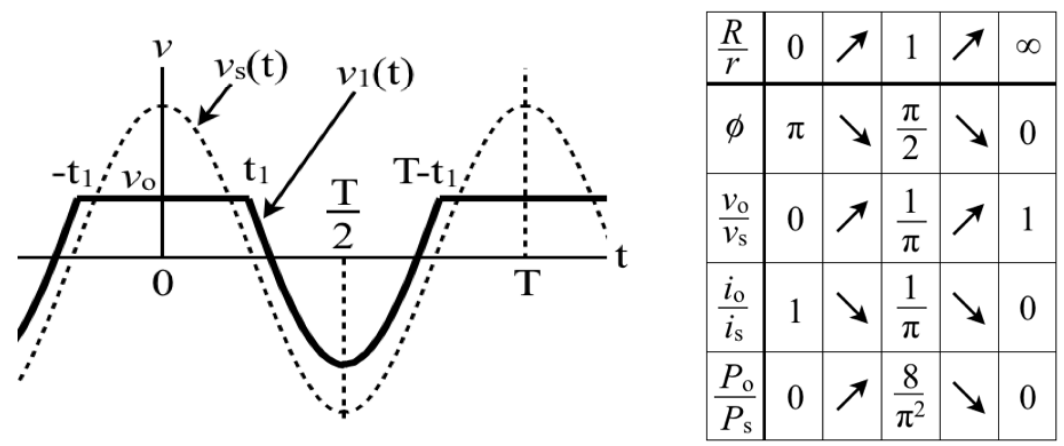

Fig. 3. Voltage time waveforms and parameters behavior as $R / r$ increases

where the circular symbol on integrals implies one cycle of $t$. The left-hand side makes zero as $v_{1}(t)$ has no DC component across an inductor. The first term of right-hand side also trivially vanishes. Since $r>0$, Eq. (6) results in

$$
\oint v_{1}(t) d t=0, \quad \oint i_{1}(t) d t=0
$$

We call them cyclostationary conditions. Integral of Eq. (4) for a cycle gives

$$
\oint i_{1}(t) d t+i_{2} T=\oint i_{d}(t) d t=\oint i_{3}(t) d t+i_{o} T
$$

As $i_{3}(t)$ has only displacement current across a capacitor, its one-cycle integral vanishes again. Since $T>0$, Eq. (8) with (4) results in

$$
i_{\mathrm{o}}=i_{2}=\frac{1}{T} \oint i_{d}(t) d t, \quad i_{1}(t)=i_{3}(t)=i_{d}(t)-\frac{1}{T} \oint i_{d}(t) d t
$$




$$
\int_{-t_{1}}^{t_{1}} v_{o} d t+\int_{t_{1}}^{T-t_{1}}\left(v_{s} \cos \omega t+r i_{2}\right) d t=0
$$

Remembering $i_{\mathrm{o}}=i_{2}, v_{\mathrm{o}}=R i_{\mathrm{o}}$, and $\omega T=2 \pi$, we can rewrite Eq. (10) as

$$
v_{s} \sin \phi=\{\phi R+(\pi-\phi) r\} i_{o}, \quad \phi=\omega t_{1}
$$

Voltage and current waveform are generally continuous at any time and any node in the circuit. This is true even with a switching diode because it transits between $\mathrm{ON}$ and OFF states always via its zero-voltage and -current origin. Imposing such continuity at transient time $t_{1}$ upon Eq. (5), we get

$$
v_{s} \cos \phi=v_{\mathrm{o}}-r i_{2}=(R-r) i_{\mathrm{o}}, \quad \phi=\omega t_{1}
$$

The quotient of Eqs. (11) to (12) yields

$$
\tan \phi-\phi=\frac{\pi r}{R-r} \quad \text { or } \quad \frac{R}{r}=\frac{\sin \phi+(\pi-\phi) \cos \phi}{\sin \phi-\phi \cos \phi}, 0<\phi<\pi
$$

where $2 \phi$ is called flow angle ranging from 0 to $2 \pi$. Feeding Eq. (13) back into (12), we finally obtain DC output current and voltage

$$
\begin{aligned}
& i_{o}=\frac{v_{s} \cos \phi}{R-r}=\frac{v_{s}}{\pi r}(\sin \phi-\phi \cos \phi) \\
& v_{\mathrm{O}}=R i_{\mathrm{O}}=\frac{R v_{s}}{\pi r}(\sin \phi-\phi \cos \phi)=\frac{v_{s}}{\pi}\{\sin \phi+(\pi-\phi) \cos \phi\}
\end{aligned}
$$

They produce output power $P_{\mathrm{o}}=i_{\mathrm{o}} v_{\mathrm{o}}$ associated with efficiency

$$
\eta=\frac{P_{\mathrm{o}}}{P_{\mathrm{s}}}=\frac{8}{\pi^{2}}(\sin \phi-\phi \cos \phi)\{\sin \phi+(\pi-\phi) \cos \phi\} \approx \frac{8}{\pi^{2}} \sin ^{3} \phi
$$

These formulas enable us to find how $\phi, v_{\mathrm{o}}, i_{\mathrm{o}}$, and $\eta$ behave as $R / r$ increases as shown in Table in Fig. 3. In particular, $\eta$ reaches its maximum

$$
\eta_{\max }=\frac{P_{\max }}{P_{\mathrm{s}}}=\frac{8}{\pi^{2}} \approx 81.1 \% \quad \text { at } \quad \frac{R}{r}=1 \quad \text { or } \quad \phi=\frac{\pi}{2}
$$

This condition should be rigorously called half-wave rectification. The rest $18.9 \%$ of input power dissipates in distortion. It means prospective room at most for efficiency improvement by reactive termination against harmonics.

\section{Single-shunt topology}

Thanks to the duality theorem, we can characterize the single-shunt topology shown in Fig. 4 by interchanging voltage/current, ON/OFF, $L / C$, and $R / r$

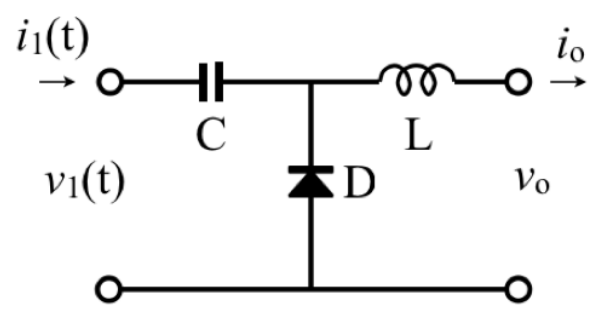

Fig. 4. Single-shunt RF rectifier 
from formulas for the single-series one. Begin with Eqs. (4) and (5) replaced by

$$
v_{1}(t)+v_{\mathrm{C}}=v_{d}(t)=v_{\mathrm{L}}(t)+v_{\mathrm{O}}
$$

(i) $\quad \mathrm{D}=\mathrm{ON} \quad v_{1}(t)=-v_{\mathrm{o}}, \quad i_{1}(t)=\frac{1}{r}\left(v_{s} \cos \omega t+v_{\mathrm{o}}\right) \quad-t_{1}<t<t_{1}$

(ii) $\mathrm{D}=\mathrm{OFF} \quad i_{1}(t)=i_{\mathrm{o}}, \quad v_{1}(t)=v_{s} \cos \omega t-r i_{\mathrm{o}} \quad t_{1}<t<T-t_{1}$

where $v_{\mathrm{C}}$ and $v_{\mathrm{L}}(t)$ stand for voltages on capacitor $C$ and inductor $L$. Then imposing the cyclostationary condition and waveform continuity described in the previous section, we reach

$$
\begin{aligned}
& \tan \phi-\phi=\frac{\pi R}{r-R} \quad \text { or } \quad \frac{R}{r}=\frac{\sin \phi-\phi \cos \phi}{\sin \phi+(\pi-\phi) \cos \phi}, \quad 0<\phi<\pi \\
& v_{\mathrm{O}}=R i_{\mathrm{O}}=\frac{R v_{s} \cos \phi}{r-R}=\frac{v_{s}}{\pi}(\sin \phi-\phi \cos \phi) \\
& i_{\mathrm{O}}=\frac{v_{s}}{\pi R}(\sin \phi-\phi \cos \phi)=\frac{v_{s}}{\pi r}\{\sin \phi+(\pi-\phi) \cos \phi\}
\end{aligned}
$$

The power performance after all remains exactly the same as described in Eqs. (16) and (17). This result agrees with the duality theorem where impedance terms are all reversed while power terms totally keep unchanged.

\section{Bridge topology}

Figure 5 shows a widely used topology for full-wave rectification. Unlike single-diode ones, we need a reactor only at the output port. This is because DC currents generated from two branches cancel each other at the input port. Capacitor $C$ acts not only for output smoothing in a passive fashion, but works for power efficiency enhancement.

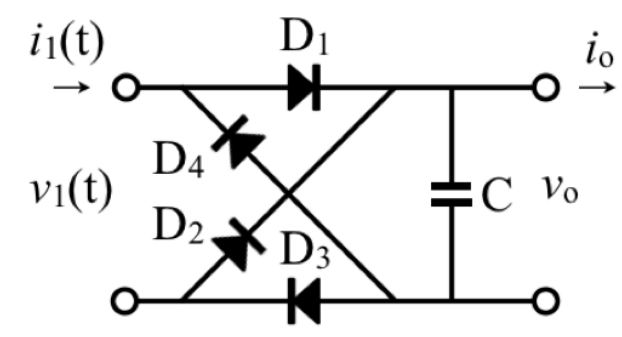

Fig. 5. Bridge RF rectifier

Since the circuit has two pairs of diodes, it looks complicated to formulate the waveforms. We actually consider four sequential states
(i) $\mathrm{D}_{1,3}=\mathrm{ON}, \mathrm{D}_{2,4}=\mathrm{OF}$
$v_{\mathrm{o}}<v_{\mathrm{s}}(t)$
$-t_{1}<t<t_{1}$
(ii) $\mathrm{D}_{1,2,3,4}=\mathrm{OFF}$
$-v_{\mathrm{O}}<v_{\mathrm{S}}(t)<v_{\mathrm{O}} \quad t_{1}<t<\mathrm{T} / 2-t_{1}$
(iii) $\mathrm{D}_{1,3}=\mathrm{OFF}, \mathrm{D}_{2,4}=\mathrm{ON}$
$v_{\mathrm{S}}(t)<-v_{\mathrm{o}}$
$\mathrm{T} / 2-t_{1}<t<\mathrm{T} / 2+t_{1}$
(iv) $\mathrm{D}_{1,2,3,4}=\mathrm{OFF}$
$-v_{\mathrm{o}}<v_{\mathrm{s}}(t)<v_{\mathrm{o}}$
$\mathrm{T} / 2+t_{1}<t<\mathrm{T}-t_{1}$

continuously taking place synchronized to RF sinusoidal stimulus $v_{\mathbf{s}}(t)$ with period $T$. Note that the two pairs cannot make ON at the same time. Defining $\phi=2 \pi t_{1} / T(0<\phi<\pi / 2)$, we call $4 \phi$ flow angle ranging from 0 to $2 \pi$. 
From Eqs. (1), (2), the above four diode states, and waveform continuity at $t_{1}$, we get voltage and current

$$
\begin{aligned}
& v_{1}(t)=\left\{\begin{array}{lc}
v_{\mathrm{S}} \cos \phi & \text { for (i) } \\
v_{s} \cos \omega t & \text { for (ii) (iv) } \\
-v_{\mathrm{s}} \cos \phi & \text { for (iii) }
\end{array}\right\}, \\
& i_{1}(t)=\left\{\begin{array}{lc}
\frac{v_{s}}{r}(\cos \omega t-\cos \phi) & \text { for (i) } \\
0 & \text { for (ii) (iv) } \\
\frac{v_{s}}{r}(\cos \omega t+\cos \phi) & \text { for (iii) }
\end{array}\right\}
\end{aligned}
$$

at the input port. Imposing the cyclostationary condition for capacitor current, we obtain flow-angle equation and output performance formulas

$$
\begin{aligned}
& \tan \phi-\phi=\frac{\pi r}{2 R}, 0<\phi<\frac{\pi}{2}, v_{\mathrm{o}}=v_{\mathrm{S}} \cos \phi, i_{\mathrm{o}}=\frac{2 v_{\mathrm{s}}}{\pi r}(\sin \phi-\phi \cos \phi) \\
& \eta=\frac{P_{\mathrm{o}}}{P_{\mathrm{S}}}=\frac{16}{\pi}(\sin \phi-\phi \cos \phi) \cos \phi=\frac{8}{\pi}(\sin 2 \phi-\phi-\phi \cos 2 \phi)
\end{aligned}
$$

To find optimum flow angle $\phi_{\text {opt }}$, solve $d \eta / d \phi=0$ on Eq. (25). We finally reach

$$
\begin{aligned}
& \tan \phi_{\mathrm{opt}}=2 \phi_{\mathrm{opt}} \quad \text { or } \quad \phi_{\mathrm{opt}} \approx 1.166 \\
& \eta_{\max }=\frac{P_{\mathrm{max}}}{P_{\mathrm{s}}} \approx 92.3 \% \text { at } \quad \frac{R}{r}=\frac{\pi}{2 \phi_{\mathrm{opt}}} \approx 1.347
\end{aligned}
$$

This is 11.2 points higher than predecessor's study [2] that deduced $\eta_{\max }=$ $81.1 \%$ without employing $C$. We therefore conclude that $C$ successfully squeezes such amount of rectified energy from diodes and brings it to the load. Factor 1.347 implies that $C$ acts to increase DC output-matching or equivalently decrease RF input-matching impedance by $34.7 \%$.

Imagine an alternative circuit in dual to the bridge shown in Fig. 5. This is done by just replacing the shunt smoothing capacitor by a series choke inductor at the output port. By this replacement, the four states become

$$
\begin{array}{lll}
\text { (i) } \mathrm{D}_{1,3}=\mathrm{ON}, \mathrm{D}_{2,4}=\mathrm{OFF} & r i_{\mathrm{o}}<v_{\mathrm{s}}(t) & -t_{1}<t<t_{1} \\
\text { (ii) } \mathrm{D}_{1,2,3,4}=\mathrm{ON} & -r i_{\mathrm{o}}<v_{\mathrm{s}}(t)<r i_{\mathrm{O}} & t_{1}<t<\mathrm{T} / 2-t_{1} \\
\text { (iii) } \mathrm{D}_{1,3}=\mathrm{OFF}, \mathrm{D}_{2,4}=\mathrm{ON} & v_{\mathrm{s}}(t)<-r i_{\mathrm{O}} & \mathrm{T} / 2-t_{1}<t<\mathrm{T} / 2+t_{1} \\
\text { (iv) } \mathrm{D}_{1,2,3,4}=\mathrm{ON} & -r i_{\mathrm{o}}<v_{\mathrm{s}}(t)<r i_{\mathrm{o}} & \mathrm{T} / 2+t_{1}<t<\mathrm{T}-t_{1}
\end{array}
$$

Due to the inductor loaded in series, the two pairs make simultaneous ON during (ii) and (iv), but not OFF at the same time. Through the same operation, we obtain

$$
\begin{aligned}
& \tan \phi-\phi=\frac{\pi R}{2 r}, 0<\phi<\frac{\pi}{2}, v_{\mathrm{o}}=\frac{2 v_{\mathrm{s}}}{\pi}(\sin \phi-\phi \cos \phi), i_{\mathrm{O}}=\frac{v_{\mathrm{s}}}{r} \cos \phi \\
& \eta_{\max }=\frac{P_{\max }}{P_{\mathrm{s}}} \approx 92.3 \% \text { at } \quad \frac{R}{r}=\frac{2 \phi_{\mathrm{opt}}}{\pi} \approx 0.742
\end{aligned}
$$

Note $R / r$ optimum 0.742 be inverse of 1.347 that appeared in Eq. (27). This is mathematically reasonable for duality. In physical effects, the choke inductor enhances the current and saves the voltage in its smoothing process. 


\section{Double-voltage topology}

Figure 6 shows a rectifier employing two diodes and two capacitors. A half cycle of input sinusoidal voltage surges through one diode, then the opposite half cycle does through another in turn. Repeating such a cycle, the voltage sums up double and charge the final capacitor. Exactly speaking, there are intervals between the two half cycles when the two diodes make simultaneously OFF. This scenario can be explained by four sequential states

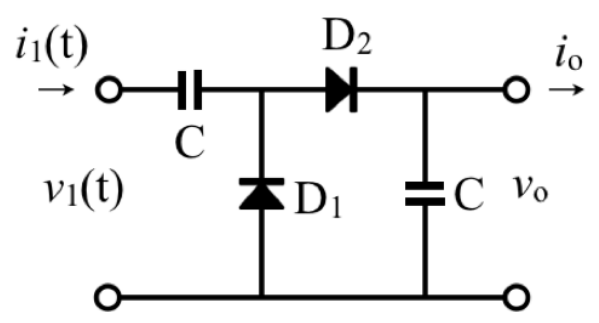

Fig. 6. Double-voltage RF rectifier
(i) $\mathrm{D}_{1}=\mathrm{OFF}, \mathrm{D}_{2}=\mathrm{ON} \quad v_{\mathrm{o}}<2 v_{\mathrm{s}}(t)$ $-t_{1}<t<t_{1}$
(ii) $\mathrm{D}_{1,2}=\mathrm{OFF}$ $-v_{\mathrm{o}}<2 v_{\mathrm{s}}(t)<v_{\mathrm{o}} \quad t_{1}<t<\mathrm{T} / 2-t_{1}$
(iii) $\mathrm{D}_{1}=\mathrm{ON}, \mathrm{D}_{2}=\mathrm{OFF}$
$2 v_{\mathrm{S}}(t)<-v_{\mathrm{O}}$ $\mathrm{T} / 2-t_{1}<t<\mathrm{T} / 2+t_{1}$
(iv) $\mathrm{D}_{1,2}=\mathrm{OFF}$
$-v_{\mathrm{o}}<2 v_{\mathrm{S}}(t)<v_{\mathrm{o}} \quad \mathrm{T} / 2+t_{1}<t<\mathrm{T}-t_{1}$

We define flow angle again as same as previous. Thanks to the continuity of the voltage and current waveforms at the input port, they are exactly same as Eq. (23). Imposing the cyclostationary condition for capacitors current, we obtain flow-angle equation and output performance formulas

$$
\begin{aligned}
& \tan \phi-\phi=\frac{2 \pi r}{R}, 0<\phi<\frac{\pi}{2}, v_{\mathrm{o}}=2 v_{\mathrm{s}} \cos \phi, i_{\mathrm{o}}=\frac{v_{\mathrm{s}}}{\pi r}(\sin \phi-\phi \cos \phi) \\
& \eta_{\max }=\frac{P_{\max }}{P_{\mathrm{s}}} \approx 92.3 \% \text { at } \frac{R}{r}=\frac{2 \pi}{\phi_{\mathrm{opt}}} \approx 5.389
\end{aligned}
$$

where $\eta$ and $\phi_{\text {opt }}$ come again from Eqs. (25) and (26). The optimum load resistance becomes four times higher than that for the first bridge topology. This result agrees with what we predicted i.e. double-voltage and half-current output from the same input power.

\section{Double-current topology}

The duality theorem enables us again to derive the formulas for the doublecurrent rectifier shown in Fig. 7. We can start by interchanging ON/OFF states, voltage/current, and source/load. The four states become

$$
\begin{array}{lll}
\text { (i) } \mathrm{D}_{1}=\mathrm{ON}, \mathrm{D}_{2}=\mathrm{OFF} & r i_{\mathrm{o}}<2 v_{\mathrm{s}}(t) & -t_{1}<t<t_{1} \\
\text { (ii) } \mathrm{D}_{1,2}=\mathrm{ON} & -r i_{\mathrm{o}}<2 v_{\mathrm{s}}(t)<r i_{\mathrm{o}} & t_{1}<t<\mathrm{T} / 2-t_{1} \\
\text { (iii) } \mathrm{D}_{1}=\mathrm{OFF}, \mathrm{D}_{2}=\mathrm{ON} & 2 v_{\mathrm{s}}(t)<-r i_{\mathrm{o}} & \mathrm{T} / 2-t_{1}<t<\mathrm{T} / 2+t_{1} \\
\text { (iv) } \mathrm{D}_{1,2}=\mathrm{ON} & -r i_{\mathrm{o}}<2 v_{\mathrm{s}}(t)<r i_{\mathrm{o}} & \mathrm{T} / 2+t_{1}<t<\mathrm{T}-t_{1}
\end{array}
$$

We define flow angle as same as previous. The voltage and current waveforms become 


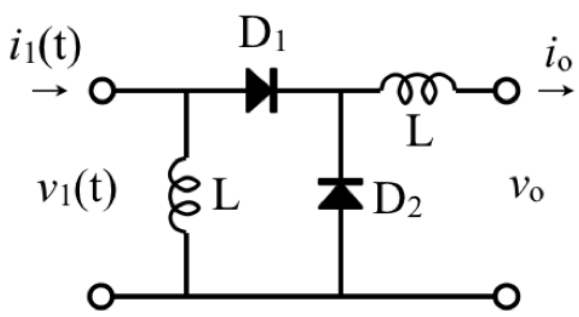

Fig. 7. Double-current RF rectifier

$$
\begin{aligned}
& v_{1}(t)=\left\{\begin{array}{lc}
v_{s}(\cos \omega t-\cos \phi) & \text { for (i) } \\
0 & \text { for (ii) (iv) } \\
v_{s}(\cos \omega t+\cos \phi) & \text { for (iii) }
\end{array}\right\}, \\
& i_{1}(t)=\left\{\begin{array}{lc}
\frac{v_{\mathrm{s}}}{r} \cos \phi & \text { for (i) } \\
\frac{v_{s}}{r} \cos \omega t & \text { for (ii) (iv) } \\
-\frac{v_{\mathrm{s}}}{r} \cos \phi & \text { for (iii) }
\end{array}\right\}
\end{aligned}
$$

at the input port. Imposing the cyclostationary condition for inductors voltage, we obtain flow-angle equation and output performance formulas

$$
\begin{aligned}
& \tan \phi-\phi=\frac{2 \pi r}{r}, 0<\phi<\frac{\pi}{2}, v_{\mathrm{o}}=\frac{v_{\mathrm{s}}}{\pi}(\sin \phi-\phi \cos \phi), i_{\mathrm{o}}=\frac{2 v_{\mathrm{s}}}{r} \cos \phi \\
& \eta_{\max }=\frac{P_{\max }}{P_{\mathrm{s}}} \approx 92.3 \% \text { at } \frac{R}{r}=\frac{\phi_{\mathrm{opt}}}{2 \pi} \approx 0.1856
\end{aligned}
$$

where $\eta$ and $\phi_{\text {opt }}$ come over again from Eqs. (25) and (26). It is worth remarking that the optimum load resistance becomes one fourth of Eq. (29), and inverse of Eq. (31). These relations sound complicated but are conveniently tabulated below.

\begin{tabular}{|c|c|c|c|c|}
\hline topology & $\begin{array}{l}\text { flow } \\
\text { angle }\end{array}$ & $\tan \phi-\phi$ & {$[R / r]_{\mathrm{opt}}$} & $\eta_{\text {max }}(\%)$ \\
\hline single ceries & \multirow{3}{*}{$2 \phi$} & $\pi r$ & \multirow{3}{*}{1.000} & \multirow{3}{*}{81.1} \\
\hline & & $R-r$ & & \\
\hline single shunt & & $\frac{\pi R}{r-R}$ & & \\
\hline bridge + capacitor & \multirow{4}{*}{$4 \phi$} & $\frac{\pi r}{2 R}$ & 1.347 & \multirow{4}{*}{92.3} \\
\hline bridge + inductor & & $\frac{\pi R}{2 r}$ & 0.742 & \\
\hline double voltage & & $\frac{2 \pi r}{R}$ & 5.389 & \\
\hline double current & & $\frac{2 \pi R}{r}$ & 0.186 & \\
\hline
\end{tabular}

\section{Conclusion}

The optimum load and maximum power efficiency formulas have been suc-

Table I. RF rectifiers theoretical properties at a glance 
cessfully derived for RF rectifiers in three pairs of topologies. The resistance ratio and flow angle play crucial roles to formulate the rectifying behaviors. The resultant formulas summarized in Table I give a clear vista to circuit designers in RF power electronics. This is much more elegant and insightful than seeking the solution by repeating nonlinear time-domain or harmonicbalance simulations. The theory can be even extended to formulation of triple- and higher-order multiple voltage or current topologies. 